\title{
Hybrid Measurement of the Energy Spectrum and Composition of Ultra-High Energy Cosmic Rays by the Telescope Array
}

\author{
Daisuke Ikeda $^{* a}$ and William Hanlon ${ }^{b}$ for the Telescope Array Collaboration \\ ${ }^{a}$ Institute for Cosmic Ray Research, University of Tokyo, Kashiwa, Chiba, Japan \\ ${ }^{b}$ High Energy Astrophysics Institute and Department of Physics and Astronomy, University of \\ Utah, Salt Lake City, UT, USA \\ E-mail: ikeda@icrr.u-tokyo.ac.jp
}

\begin{abstract}
The energy spectrum and mass composition of Ultra-High Energy Cosmic Rays (UHECRs) above $10^{18.2} \mathrm{eV}$ measured by Telescope Array (TA) are presented. TA is the largest UHECR observatory in the northern hemisphere, located in the west desert of Utah, USA. It consists of 507 Surface Detectors (SDs) and three fluorescence detectors (FDs) to observe UHECRs by those two types of detectors simultaneously, in so called hybrid observation. In hybrid analysis, a reconstruction of the longitudinal development of the air shower observed by FD with the information at the ground observed by SD, can measure the energy of the primary cosmic ray and the depth of the shower maximum precisely. The performance of the analysis, energy spectrum and mass composition of UHECR measured by TA hybrid data is discussed.
\end{abstract}

35th International Cosmic Ray Conference - ICRC2017

10-20 July, 2017

Bexco, Busan, Korea

\footnotetext{
*Speaker.
} 


\section{Introduction}

The Telescope Array (TA)[1] is the largest Ultra-High Energy Cosmic Ray (UHECR) observatory in the northern hemisphere to search for the origin of UHECR and the mechanisms of production, acceleration at the sources and propagation in the inter-galactic space. The experiment operates in hybrid mode using both of the fluorescence detector (FD)[2] and surface detector (SD)[3]. The SD array consists of 507 particle detectors which have two layers of $3 \mathrm{~m}^{2}$ plastic scintillators to detect the secondly ground level particles produced by the primary cosmic ray. These are arranged as a grid with $1.2 \mathrm{~km}$ spacing which covers an area of $\sim 760 \mathrm{~km}^{2}$. Surrounding the SD array three FD stations have been installed to measure the longitudinal development of air showers by using the fluorescence photons emitted by atmospheric molecules excited by the secondly particles. The Black Rock Mesa (BR) and Long Ridge (LR) stations are located to the southeast and southwest of the TA SD array, respectively. Each station has 12 telescopes which consists of a segmented mirror of $3.3 \mathrm{~m}$ diameters and a camera consisting of 256 PMTs.

The energy spectrum and mass composition are important keys to understanding the origin of the UHECR. The FD observes the longitudinal development of the air shower directly. By using this observation technique, the energy of UHECR is measured by a calorimetric way which is less dependent on the high energy interaction model. In addition, the depth at the maximum shower development, $X_{\max }$, is known to be a sensitive parameter for the mass composition of the UHECR. Therefore, the FD has an important roll in determining the energy scale and mass composition in the TA.

In addition, information on the ground level shower measured by the SDs helps improve the reconstruction for the FD events. The hybrid events, observed both by FD and SD, can reconstruct the UHECR with the better resolution than the FD monocular reconstruction.

In this paper, we present the performance of the developed hybrid reconstruction. The measured energy spectrum and composition of UHECR from hybrid events observed by two FDs (BR and LR) and SDs are also presented.

\section{Hybrid analysis}

At the start of the reconstruction procedure, the shower geometry, direction and arrival position on the ground, are reconstructed by using the timing of the arrival photons and pointing direction at the PMTs in the telescope camera:

$$
T_{\text {exp }, i}=T_{\text {core }}+\frac{\sin \psi-\sin \alpha_{i}}{c \sin \left(\psi+\alpha_{i}\right)} R_{\text {core }}
$$

where $T_{\exp , i}$ and $\alpha_{i}$ are the expected timing and elevation angle in the SDP for the $i$-th PMT, respectively, $T_{\text {core }}$ is the time when the air shower reached the ground, $R_{\text {core }}$ is the distance from the FD station to the core, and $\psi$ is the elevation angle of the air shower in the SDP (Figure 1). The timing information of the SD near the core is also added to this function.

Then the longitudinal development of the air shower is reconstructed. In order to include the effect of the detailed sensitivity of the telescope (non-uniformity of the PMT surface, shadowing by the telescope structure, spot size on the camera and so on), an inverse Monte-Carlo (IMC) technique 


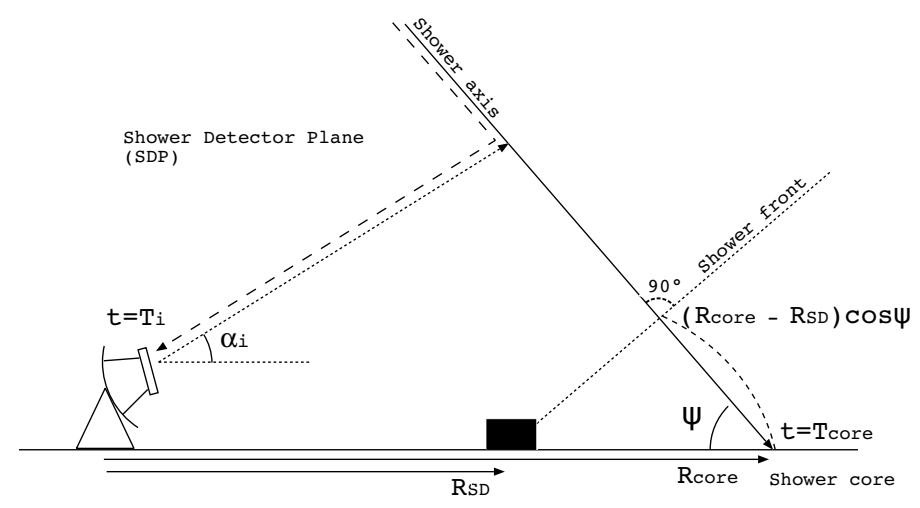

Figure 1: Parameters using for the time fit along the Shower Detector Plane (SDP).

is used in this process. This process compares the observed signals in each PMT between data and MC generated by the Gaisser-Hillas function:

$$
N\left(X ; X_{\max }, X_{0}, \Lambda\right)=N_{\max }\left(\frac{X-X_{0}}{X_{\max }-X_{0}}\right)^{\left(X_{\max }-X_{0}\right) / \Lambda} e^{\left(X_{\max }-X\right) / \Lambda},
$$

where $X$ is the atmospheric depth, $X_{\max }$ is the depth at the shower maximum, $\Lambda$ is the interaction length of the shower particles, and $X_{0}$ is the offset of $X$. Here the $X_{0}$ and $\Lambda$ are fixed to $-60 \mathrm{~g} / \mathrm{cm}^{2}$ and $70 \mathrm{~g} / \mathrm{cm}^{2}$, respectively. The shower $X_{\max }$ is obtained from the above process, and the energy of the UHECR is determined by the integration of the fitted Gaisser-Hillas function with the correction of the "missing" energy which corresponds to the invisible energy mainly from the neutrinos. The details of the analysis procedure for the hybrid events are described in [4].

To ensure reconstruction quality, we only accept events that satisfy specific criteria. For the spectrum analysis, the criteria are as follows;(1) the number of PMTs used in the reconstruction is greater than 20, (2) the zenith angle of the reconstructed shower axis is less than 55 degrees, (3) the shower core is inside the edges of the SD array, (4) the angle between the reconstructed shower axis and the telescope is greater than 20 degrees, (5) $X_{\max }$ is observed in the field of view of the telescopes. For the mass composition analysis, we applied additional criteria;(6) the $\chi^{2}$ for the geometry fit is less than $10,(7)$ the $\chi^{2}$ for the longitudinal profile fit is less than 5, (8) the track length is greater than 10 degrees, (9) the $\psi$ angle is less than 130 degrees, (10) the time extent of the track is more than $7 \mu \mathrm{s}$.

In this analysis, we use the fluorescence yield model reported by Kakimoto et al. [5] with the spectrum measured by the FLASH experiment [6]. The atmospheric conditions (pressure, temperature and humidity for each height) are reported for each height from the Global Data Assimilation System (GDAS) [7].

The performance of the reconstruction, the aperture for the energy spectrum and expected $\mathrm{X}_{\max }$ profile are evaluated by using a CORSIKA based air shower simulator [8] with the detector simulation both for SD and FD. Here we used proton and iron primary particles with the QGSJETII-03 hadronic interaction model. By using this MC set, we find the resolutions to be 0.9 degrees for the arrival direction, $7 \%$ for the energy and $20 \mathrm{~g} / \mathrm{cm}^{2}$ for the $\mathrm{X}_{\max }$. 


\section{Energy spectrum}

The energy spectrum of the UHECR is obtained from 8 years of observation data from May 11 2008 to May 11 2016. The total live time with the clear nights is about 4500 hours. The number of remaining events that pass above analysis procedure is 3209 above $10^{18.2} \mathrm{eV}$. The obtained energy spectrum is shown in Figure 2.

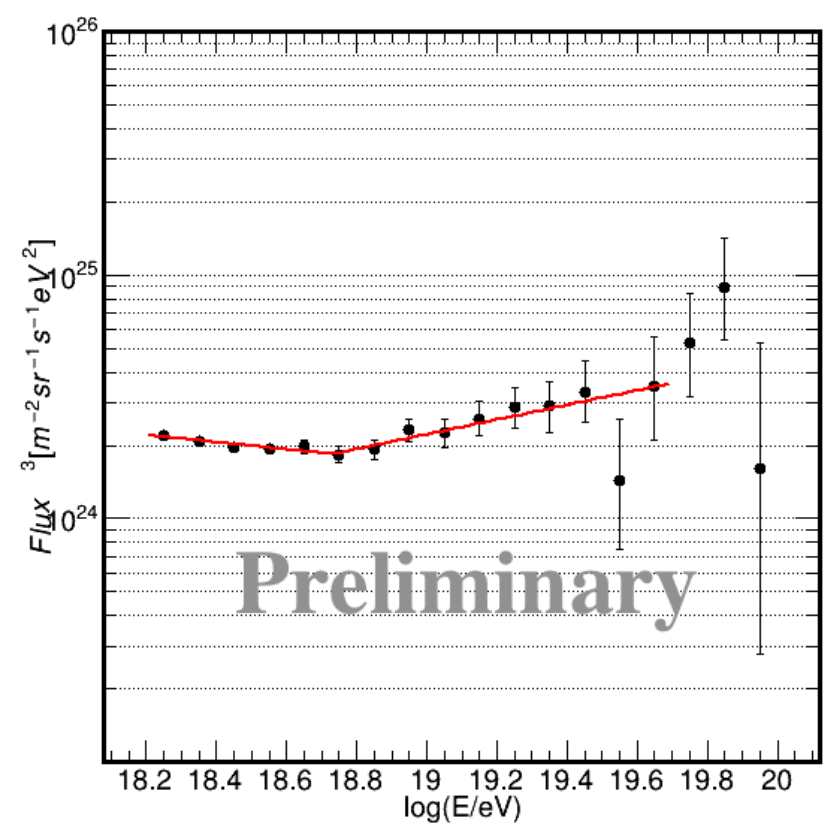

Figure 2: The energy spectra multiplied by $\mathrm{E}^{3}$. The black points show the energy spectrum obtained from the 8 years BR/LR hybrid events. The red line is the fitted broken power law function.

\section{Mass composition}

In order to obtain information on the UHECR composition, the mean $\mathrm{X}_{\max }$ as a function of energy is used. The expected mean $\mathrm{X}_{\max }$ for each composition is obtained from the MC events which are reconstructed using the same procedure as the observed data. To verify this analysis, we have prepared two independent analysis programs. This allows us to understand the systematic error of the $\mathrm{X}_{\max }$ analysis. The comparison of the observed mean $\mathrm{X}_{\max }$ above $10^{18.2} \mathrm{eV}$ between data and MC for the two analyses is shown in Figure 3. The obtained mean $\mathrm{X}_{\max }$ is consistent with a light primary composition model.

\section{Summary}

The energy spectrum and mean $\mathrm{X}_{\max }$ of UHECR above $10^{18.2} \mathrm{eV}$ obtained from 8 years of TA BR/LR hybrid data are presented. The mass composition measurement performed by observing the depth of shower maximum $\left(\mathrm{X}_{\max }\right)$ is consistent with a light primary model. 


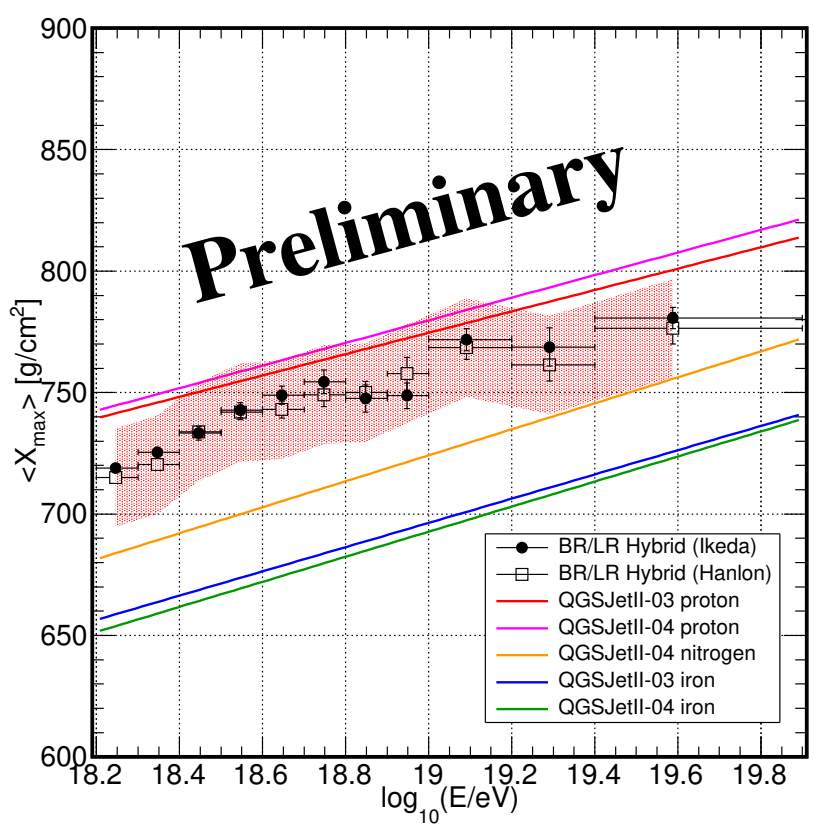

Figure 3: The mean $X_{x \max }$ as a function of the primary energy using TA BR/LR hybrid data. The band shows the systematic uncertainties of $20.3 \mathrm{~g} / \mathrm{cm}^{2}$.

\section{Acknowledgements}

The Telescope Array experiment is supported by the Japan Society for the Promotion of Science through Grants-in-Aid for Scientific Research on Specially Promoted Research (21000002) "Extreme Phenomena in the Universe Explored by Highest Energy Cosmic Rays" and for Scientific Research (19104006), and the Inter-University Research Program of the Institute for Cosmic Ray Research; by the U.S. National Science Foundation awards PHY-0307098, PHY-0601915, PHY-0649681, PHY-0703893, PHY-0758342, PHY-0848320, PHY-1069280, PHY-1069286, PHY1404495 and PHY-1404502; by the National Research Foundation of Korea (2015R1A2A1A01006870, 2015R1A2A1A15055344, 2016R1A5A1013277, 2007-0093860, 2016R1A2B4014967); by the Russian Academy of Sciences, RFBR grant 16-0200962a (INR), IISN project No. 4.4502.13, and Belgian Science Policy under IUAP VII/37 (ULB). The foundations of Dr. Ezekiel R. and Edna Wattis Dumke, Willard L. Eccles, and George S. and Dolores Doré Eccles all helped with generous donations. The State of Utah supported the project through its Economic Development Board, and the University of Utah through the Office of the Vice President for Research. The experimental site became available through the cooperation of the Utah School and Institutional Trust Lands Administration (SITLA), U.S. Bureau of Land Management (BLM), and the U.S. Air Force. We appreciate the assistance of the State of Utah and Fillmore offices of the BLM in crafting the Plan of Development for the site. We also wish to thank the people and the officials of Millard County, Utah for their steadfast and warm support. We gratefully acknowledge the contributions from the technical staffs of our home institutions. An allocation of computer time from the Center for High Performance Computing at the University of 
Utah is gratefully acknowledged.

\section{References}

[1] H. Kawai et al. , J. Phys. Soc. Jpn. Suppl. A 78 108-113, (2009)

[2] H. Tokuno et al. , Nucl. Instrum. Meth. A 676, 54-65 (2012)

[3] T. Abu-Zayyad et al. , Nucl. Instrum. Meth. A 689, $87-97$ (2012)

[4] T. Abu-Zayyad et al., Astropart. Phys. 61 (2015) 93-101.

[5] F. Kakimoto et al. , Nucl. Instrum. Meth. A 372 (1996) 527-533.

[6] R.U. Abassi et al. , Astropart. Phys. 29 (2008) 77-86.

[7] www.ncdc.noaa.gov/data-access/model-data/model-datasets/global-data-assimilation-system-gdas

[8] B.T. Stokes et al. , Astropart. Phys. 35 (2012) 759-766. 